\section{BRAZIULIAN JOURNAL}

OF MEDICAL AND BIOLOGICAL RESFARCH

www.bjournal.com.br
ISSN 0100-879X

Volume 42 (8) 692-775 August 2009

CLINICAL INVESTIGATION

Braz J Med Biol Res, August 2009, Volume 42(8) 744-749

How frequently should a patient taking amiodarone be screened for thyroid dysfunction?

A. Pazin-Filho, A.M.X. de Jesus, P.K.R. Magalhães, L.H. Melato, D. Campos, B.C. Maciel and L.M.Z. Maciel

The Brazilian Journal of Medical and Biological Research is partially financed by
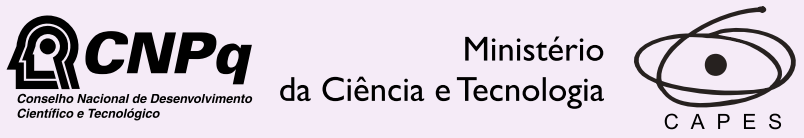

Ministério da Educação

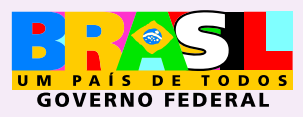

Institutional Sponsors 


\title{
How frequently should a patient taking amiodarone be screened for thyroid dysfunction?
}

\author{
A. Pazin-Filho' ${ }^{1}$ A.M.X. de Jesus' ${ }^{2}$, P.K.R. Magalhães ${ }^{2}$, L.H. Melato ${ }^{3}$, \\ D. Campos ${ }^{4}$, B.C. Maciel ${ }^{3}$ and L.M.Z. Maciel ${ }^{2}$ \\ 'Divisão de Emergências Clínicas, 'Divisão de Endocrinologia e Metabologia, ${ }^{3}$ Divisão de Cardiologia, \\ Departamento de Clínica Médica, ${ }^{4}$ Departamento de Medicina Social, \\ Faculdade de Medicina de Ribeirão Preto, Universidade de São Paulo, \\ Ribeirão Preto, SP, Brasil
}

\begin{abstract}
Amiodarone-induced thyroid dysfunction (AITD) is a common complication of amiodarone therapy and its prevalence varies according to iodine intake, subclinical thyroid disorders and the definition of AITD. There is no consensus about the frequency of screening for this condition. We evaluated 121 patients on chronic regular intake of amiodarone (mean intake $=248.5 \pm 89$ $\mathrm{mg}$; duration of treatment $=5.3 \pm 3.9$ years, range $=0.57-17$ years) and with stable baseline cardiac condition. Those with no AITD were followed up for a median period of 3.2 years (range: 0.6-6.7) and the incidence rate of AITD, defined by clinical and laboratorial findings as proposed by international guidelines, was obtained (62.8 per 1000 patients/year). We applied the Cox proportional hazard model to adjust for potential confounding factors and used sensitivity analysis to identify the best screening time for follow-up. We detected thyroid dysfunction in $59(48.7 \%)$ of the 121 patients, amiodarone-induced hypothyroidism in $50(41.3 \%)$ and hyperthyroidism in $9(7.5 \%)$. Compared with patients without AITD, there was no difference regarding dosage or duration of therapy, heart rhythm disorder or baseline cardiac condition. During the follow-up of the 62 patients without AITD at baseline evaluation, 11 developed AITD (interquartile range, IR: 62.8 (95\% Cl: 31.3-112.3) cases per 1000 patients/year), 9 of them with hypothyroidism - IR: 11.4 (95\% Cl: 1.38-41.2), and 2 hyperthyroidism - IR: 51.3 (95\%Cl: 23.4-97.5). Age, gender, dose, and duration of treatment were not significant after adjustment. During the first 6 months of follow-up the incidence rate for AITD was 39.3 (9.2-61.9) cases per 1000 patients/year. These data show that AITD is quite common, and support the need for screening at 6-month intervals, unless clinical follow-up dictates otherwise or further information regarding the prognosis of untreated subclinical AITD is available.
\end{abstract}

Key words: Amiodarone; Chagas' disease; Thyroid dysfunction; Antiarrhythmic drug

\section{Introduction}

Amiodarone is a diiodinated benzofuran derivative widely used for its antiarrhythmic properties (1). Recently, it has been included in the American HeartAssociation guidelines for cardiac arrest and emergency care (2). Although it has proved to be a very effective drug for the treatment of cardiac arrhythmias, its chronic use is associated with significant side effects. Among these, thyroid dysfunction is a common manifestation (3), even when a low daily dose regime of amiodarone is used. The exact prevalence and pathogenesis of amiodarone-induced thyrotoxicosis (AIT) or hypothyroidism $(\mathrm{AlH})$ are unknown, but they are associ- ated with several factors. First, there is a very important relationship with iodine intake, so that in regions where an inadequate low intake occurs, a greater prevalence of AIT and goiter will be detected, while in areas where a sufficient iodine intake is observed, a greater prevalence of AlH can be detected (4). Second, the criteria used to define AIT have to be taken into consideration, since common symptoms of thyroid dysfunction can be masked by the effects of amiodarone, so that thyroid dysfunction can only be diagnosed by active thyroid hormone evaluation (1). Third, the time when the hormone level is determined

Correspondence: L.M.Z. Maciel, Departamento de Clínica Médica, FMRP, USP, Av. Bandeirantes, 3900, 14049-900 Ribeirão Preto, SP, Brasil. Fax: +55-16-3602-2666. E-mail: Imzmacie@fmrp.usp.br

Research supported by CNPq (Grant PIBIC to A.M.X. de Jesus). Received October 24, 2008. Accepted June 8, 2009. 
is very important since fluctuation can occur, especially at the beginning of treatment (4). Fourth, AIT can be caused by direct (drug effect on the thyroid gland inducing a destructive thyroiditis) or indirect (exacerbating subclinical conditions such as pre-existing or "latent" thyroid disease by a multinodular goiter ou Graves' disease) effects of the drug $(1,4,5)$. It should be mentioned that in most of the previously published studies the differentiation between these mechanisms of AIT was not available.

In view of these considerations, screening for thyroid function has been recommended in several guidelines (4,68 ), but there is no consensus concerning the recommended frequency of this screening process. Intervals varying from 3 months to more than 1 year have been recommended. Thus, the objective of the present study was to evaluate the incidence of thyroid dysfunction in cardiac patients using amiodarone on regular basis for more than one year, after excluding prevalent thyroid dysfunction. The results of this evaluation should be helpful for determining the most appropriate interval for amiodarone-induced thyroid dysfunction (AITD) screening during chronic use of amiodarone.

\section{Material and Methods}

\section{Study population and exposure}

A total of 121 patients ( $43.8 \%$ males; $58.3 \pm 13.2$ years old) using amiodarone for cardiac rhythm disorders were selected consecutively among patients who sought medical attention between 2000 and 2001 at the cardiology outpatient clinic of the University Hospital, Medical School of Ribeirão Preto, University of São Paulo, Ribeirão Preto, SP, Brazil. The patients had been using amiodarone on a daily basis for a median period of 30 months (interquartile range: 16-56), with an average dose of less than $250 \mathrm{mg} /$ day. They did not have any known thyroid disease before starting amiodarone and had clinically stable heart disease at the time when they were invited to participate in the study and their thyroid function hormones were evaluated. Demographic and clinical data regarding age, gender, amiodarone dose, type of cardiac rhythm disorder, and baseline cardiovascular disease were collected for all patients.

The patients were submitted to clinical and laboratory evaluation by an endocrine disease specialist for characterization of their thyroid function status. Patients who were found to have any thyroid dysfunction (50 patients with hypothyroidism and 9 patients with hyperthyroidism) were excluded from follow-up and referred to the endocrinology outpatient clinic for follow-up and treated accordingly. Of the remaining 62 patients presenting normal thyroid function at baseline, 9 were excluded from analysis since amiodarone was discontinued based on clinical decision. The remaining 53 patients were followed for a mean of 3.2 years (median 3.2; range 0.6-16.5) for thyroid dysfunction at 6-month intervals. The protocol was approved by the Ethics Committee of Faculdade de Medicina de Ribeirão Preto,
Universidade de São Paulo (HCRP No. 8557/2004), and written informed consent was obtained from each patient.

\section{Thyroid function evaluation}

In addition to a complete medical history and physical examination emphasizing thyroid-related signs and symptoms, clinical evaluation included the determination of serum thyrotrophin (TSH), free thyroxine (FT4), free triiodothyroxine (FT3), and thyroid auto-antibodies. TSH, FT4, and FT3 were measured by a chemiluminescent method (Immulite 2000, DPC Cirrus Inc., USA) with sensitivity of $0.02 \mu \mathrm{U} /$ $\mathrm{mL}, 0.15 \mathrm{ng} / \mathrm{dL}$, and $1.0 \mathrm{pg} / \mathrm{mL}$, respectively. The intraassay coefficient of variation (CV) was $3.8,5.2$ and $3.5 \%$, and interassay $\mathrm{CV}$ was $3.5,14$, and $14.6 \%$, respectively. Anti-peroxidase (anti-TPO) antibodies were measured by a chemiluminescent method (Immulite 2000, DPC Cirrus Inc.) with an intra-assay CV of $2.3 \%$ and an interassay CV of $10.5 \%$. Reference values were $0.3-4.0 \mathrm{mU} / \mathrm{L}$ for TSH, $1.5-$ $4.1 \mathrm{pg} / \mathrm{mL}$ for FT3, and 0.7-1.7 ng/dL for FT4, and anti-TPO values above $35 \mathrm{U} / \mathrm{L}$ were considered to be positive.

\section{Definition of thyroid dysfunction}

Hormone determinations and/or clinical evidence of thyroid dysfunction were used to identify patients presenting thyroid dysfunction. Patients with TSH lower than 0.1 $\mathrm{mU} / \mathrm{L}$ were considered to have hyperthyroidism, which was defined as subclinical if FT3 and FT4 were normal and clinical if FT3 and/or FT4 were high. Patients with TSH equal to or higher than $4.5 \mathrm{mU} / \mathrm{L}$ were considered to have hypothyroidism, which was defined as subclinical if FT3 and FT4 were normal and clinical if FT3 and/or FT4 were low. The criterion used to diagnose hyperthyroidism was based on the guidelines of the American Thyroid Association, suggesting that virtually all cases of hyperthyroidism encountered in clinical practice are accompanied by a serum TSH concentration lower than $0.1 \mathrm{mU} / \mathrm{L}$ and not by TSH just below the normal range (9).

\section{Statistical analysis}

Demographic and clinical characteristics were compared using chi-square tests or non-parametric tests (KruskalWallis or Mann-Whitney) as applicable. Crude AITD incidence rates and $95 \%$ confidence intervals $(95 \% \mathrm{Cl})$ were calculated using time-to-event methods. The proportion of individuals remaining free of incident AITD at any time during follow-up was calculated using the Kaplan-Meier method. For all survival analyses, the follow-up time was defined as the period from entry into the study to AITD diagnosis or up to the time an individual left the study. We used Cox proportional hazard models to adjust for age (years), gender, drug dose, and duration of treatment. We also performed a sensitivity analysis, limiting the follow-up to 6 months and 1 year to evaluate the impact of time on the AITD incidence rate. All analyses were conducted using the Stata version 9.2 software (10). 


\section{Results}

Of the 121 patients studied, 59 were diagnosed with AITD at the beginning of the study indicating a prevalence rate of $48.7 \%$ : 50 with $\mathrm{AlH}(41.3 \%)$ and 9 (7.5\%) with AIT. Comparison of these 59 patients who presented thyroid disfunction at the beginning of the study with the 62 euthyroid patients can be found in Table 1. Even though the patients with AIT tended to be older than the AIH and euthyroid patients, no significant difference was found between groups. There was a greater prevalence of female gender among participants with AITD. Amiodarone dose, treatment duration and prevalence of cardiac rhythm disorder did not differ between groups. Of interest, Chagas' cardiomyopathy was highly prevalent in our population using amiodarone $(43.4 \%)$, but no demographic or clinical difference could be demonstrated when we compared Chagas' disease patients to patients with other cardiomyopathies or presenting any other baseline cardiac disease. Anti-TPO levels were higher in the hypothyroid group.

Of the 50 patients diagnosed with $\mathrm{AlH}$, only $16(32 \%)$ had clinical signs and symptoms, while most AIT patients had overt clinical disease $(77.7 \%)$. There was no difference in the demographic and clinical characteristics regarding patients with AlH when we compared those presenting clinical manifestations with those who had subclinical disease (Table 2), except for TSH and FT4 levels.

During the follow-up of patients who were euthyroid at baseline (62; 51.2\%), 9 discontinued the use of amiodarone and of the remaining 53 patients, 11 developed AITD, 9 were diagnosed as AIH and 2 as AIT. The incidence rate of AITD was 62.8 per 1000 patients/year (95\% Cl: 31.3-112.3),

Table 1. Demographic, clinical and laboratory baseline evaluation according to thyroid function status of patients taking amiodarone.

\begin{tabular}{|c|c|c|c|c|}
\hline & Euthyroid & $\mathrm{AlH}$ & AIT & $\mathrm{P}$ \\
\hline $\mathrm{N}(\%)$ & $62(51.2 \%)$ & $50(41.3 \%)$ & $9(7.5 \%)$ & \\
\hline Age (years) & $57.9 \pm 13.7$ & $57.6 \pm 11.9$ & $65.2 \pm 16.9$ & \\
\hline Gender (female/ male, \% female) & $27 / 35(39.7 \%)$ & $34 / 16(50 \%)$ & $7 / 2(77.7 \%)$ & $0.014^{a}$ \\
\hline \multicolumn{5}{|c|}{ Time of amiodarone intake prior to enrollment (months) } \\
\hline Mean \pm SD & $46.0 \pm 49.3$ & $49.2 \pm 47.3$ & $40.5 \pm 51$ & \\
\hline Median (range) & $31(6-300)$ & $28(7-161)$ & $15(8-164)$ & \\
\hline \multicolumn{5}{|l|}{ Dose, N (\%) } \\
\hline$\leq 250 \mathrm{mg}$ & $42(67.7 \%)$ & $35(70 \%)$ & $8(88.9 \%)$ & \\
\hline$>250 \mathrm{mg}$ & $20(32.3 \%)$ & $15(30 \%)$ & $1(11.1 \%)$ & \\
\hline \multicolumn{5}{|l|}{ Cardiac rhythm disorder, N (\%) } \\
\hline SVT & $29(46.7 \%)$ & $18(36 \%)$ & $4(44.4 \%)$ & \\
\hline VT & $26(41.9 \%)$ & $30(60 \%)$ & $4(44.4 \%)$ & \\
\hline IND & $7(11.2 \%)$ & $2(4 \%)$ & $1(11.1 \%)$ & \\
\hline Baseline cardiac condition, N (\%) & & & & $0.032^{\mathrm{a}}$ \\
\hline CAD & $11(17.7 \%)$ & $6(12 \%)$ & $1(11.1 \%)$ & \\
\hline Cardiomyopathy & $42(67.7 \%)$ & $27(54 \%)$ & $4(44.4 \%)$ & \\
\hline Valvular disease & $7(11.3 \%)$ & $12(24 \%)$ & $1(11.1 \%)$ & \\
\hline Other & $2(3.2 \%)$ & $5(10 \%)$ & $3(33.3 \%)$ & \\
\hline Overt clinical disease, $\mathrm{N}(\%)$ & 0 & $16(32 \%)$ & $7(77.7 \%)$ & \\
\hline \multicolumn{5}{|c|}{ Laboratory evaluation of thyroid function (mean $\pm \mathrm{SD}$ ) } \\
\hline TSH (mU/L) & $1.5 \pm 0.92$ & $20.61 \pm 28.19$ & $0.06 \pm 0.04$ & $<0.001^{b}$ \\
\hline FT3 (pg/mL) & $2 \pm 0.55$ & $2.48 \pm 0.67$ & $2.75 \pm 1.08$ & $0.003^{b}$ \\
\hline FT4 (ng/mL) & $1.45 \pm 0.36$ & $0.98 \pm 0.44$ & $2.06 \pm 0.31$ & $0.001^{\mathrm{b}}$ \\
\hline Anti-TPO (U/L) & $22 \pm 14.01$ & $528.21 \pm 1267.26$ & $29.22 \pm 14.47$ & $0.045^{b}$ \\
\hline
\end{tabular}

Reference values: thyrotrophin $(\mathrm{TSH})=0.3-4.0 \mathrm{mU} / \mathrm{L}$; free triiodothyroxine $(\mathrm{FT} 3)=1.5-4.1 \mathrm{pg} / \mathrm{mL}$; free thyroxine $(\mathrm{FT} 4)=0.7-1.7 \mathrm{ng} /$ $\mathrm{mL}$; antithyroid peroxidase antibody $($ anti-TPO) $=>35 \mathrm{U} / \mathrm{L}$. AlH $=$ amiodarone-induced hypothyroidism; AIT $=$ amiodarone-induced thyrotoxicosis; SVT = supraventricular tachycardia; VT = ventricular tachycardia; IND = indeterminate; CAD = coronary artery disease. Data were analyzed statistically by the chi-square test (a) for categorical variables and by the Kruskal-Wallis test (b) for continuous variables, as appropriate. 
Table 2. Demographic, clinical and laboratory baseline evaluation of patients for amiodarone-induced hypothyroidism according to clinical manifestation status.

\begin{tabular}{|c|c|c|c|}
\hline & Subclinical & Clinical & $\mathrm{P}$ \\
\hline$N(\%)$ & $34(68 \%)$ & $16(32 \%)$ & \\
\hline Age (years) & $57.0 \pm 10.5$ & $58.8 \pm 14.8$ & \\
\hline Gender (female/male, \% female) & $24 / 10(70.6 \%)$ & $10 / 6(62.5 \%)$ & \\
\hline \multicolumn{4}{|c|}{ Time of amiodarone intake prior to enrollment (months) } \\
\hline Mean \pm SD & $52.0 \pm 45.5$ & $42.8 \pm 52.4$ & \\
\hline Median (range) & $33.5(7-156)$ & $16(9-161)$ & \\
\hline \multicolumn{4}{|l|}{ Dose, N (\%) } \\
\hline$\leq 250 \mathrm{mg}$ & $24(70.6 \%)$ & $11(68.7 \%)$ & \\
\hline$>250 \mathrm{mg}$ & $10(29.4 \%)$ & $5(31.3 \%)$ & \\
\hline \multicolumn{4}{|l|}{ Cardiac rhythm disorder, N (\%) } \\
\hline SVT & $12(35.3 \%)$ & $6(37.5 \%)$ & \\
\hline VT & $20(58.8 \%)$ & $10(62.5 \%)$ & \\
\hline IND & $2(5.9 \%)$ & $0(0 \%)$ & \\
\hline \multicolumn{4}{|l|}{ Cardiac baseline condition, $\mathrm{N}(\%)$} \\
\hline CAD & $4(11.7 \%)$ & $2(12.5 \%)$ & \\
\hline Cardiomyopathy & $17(50.0 \%)$ & $10(62.5 \%)$ & \\
\hline Valvular disease & $10(29.4 \%)$ & $2(12.5 \%)$ & \\
\hline Other & $3(8.8 \%)$ & $2(12.5 \%)$ & \\
\hline \multicolumn{4}{|c|}{ Laboratory evaluation of thyroid function (mean \pm SD) } \\
\hline TSH (mU/L) & $7.57 \pm 4.13$ & $48.3 \pm 36.8$ & $<0.001^{\mathrm{a}}$ \\
\hline FT3 $(\mathrm{pg} / \mathrm{mL})$ & $2.5 \pm 0.7$ & $2.4 \pm 0.5$ & \\
\hline FT4 (ng/mL) & $1.1 \pm 0.3$ & $0.4 \pm 0.1$ & \\
\hline Anti-TPO (U/L) & $729.2 \pm 1577.6$ & $203.5 \pm 288.5$ & $<0.001^{\mathrm{a}}$ \\
\hline
\end{tabular}

Reference values: thyrotrophin $(\mathrm{TSH})=0.3-4.0 \mathrm{mU} / \mathrm{L}$; free triodothyroxine $(\mathrm{FT} 3)=1.5-4.1 \mathrm{pg} / \mathrm{mL}$; free thyroxine $(\mathrm{FT} 4)=0.7-1.7 \mathrm{ng} /$ $\mathrm{mL}$; antithyroid peroxidase antibody $($ anti-TPO) $=>35 \mathrm{U} / \mathrm{L}$. SVT = supraventricular tachycardia; VT = ventricular tachycardia; IND = indeterminate; $C A D=$ coronary artery disease. Data were analyzed statistically by the Kruskal-Wallis test (a) for continuous variables and by the chi-square test for categorical variables.

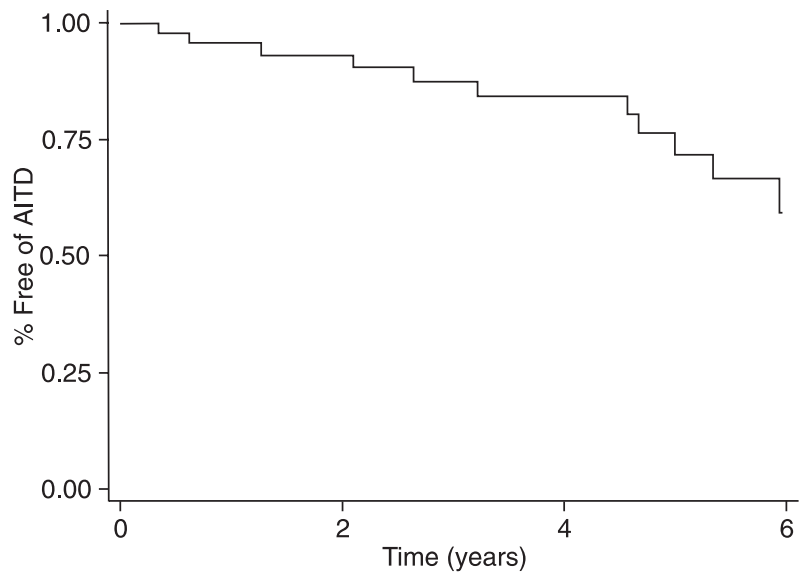

Figure 1. Kaplan-Meier survival curve for the 62 patients without thyroid dysfunction at baseline who developed amiodaroneinduced thyroid dysfunction (AIDT) over a mean period of 3.2 years of follow-up. the rate for $\mathrm{AlH}$ was 51.3 per 1000 patients/year $(95 \% \mathrm{Cl}$ : 23.4-97.5), and the rate for AIT was 11.4 (95\%Cl: $1.38-$ 41.2). Figure 1 provides the Kaplan-Meier curve for AITD. Adjusting for age, gender, dose, and duration of treatment did not change our results. When we limited the followup time to 6 months in our sensitivity analysis, the AITD incidence rate was 39.3 per 1000 patients/year $(95 \% \mathrm{Cl}$ : 9.2-61.9), not very different from the 1 year follow-up 41.7 per 1000 patients/year $(95 \% \mathrm{Cl}: 5.2-150.8)$.

\section{Discussion}

The prevalence of AITD varies according to iodine intake supplementation, presence of subclinical thyroid disorders and AITD definition $(1,4,7)$. Our results show a high prevalence of AlH (41.3\%), and a low prevalence of AIT (7.5\%) probably because our region is an area of sufficient iodine intake. For areas such as ours with iodine sufficiency, a prevalence of $25 \%$ for $\mathrm{AlH}$ and a $2 \%$ prevalence of AIT 
induced by amiodarone has been reported (11), lower values than those observed in the present study. These findings may reflect differences between populations, such as a higher prevalence of females in our group. They may also reflect the fact that there was no standard protocol in our institution for routine thyroid function screening for patients taking amiodarone prior to 2001. In addition, the prevalence in our study was much higher than that for the general population, which ranges from 7 to $10 \%$, using TSH levels for clinical diagnosis.

One of the confounding factors for studying AITD prevalence is the lack of data considering baseline thyroid function for persons who start amiodarone. Specifically among patients with hypothyroidism, subclinical autoimmune disorders could be responsible for hypothyroidism during follow-up or may be precipitated by amiodarone intake. It was impossible to evaluate this among our patients with hypothyroidism at baseline, even though the higher anti-TPO levels in this group could have suggested this possibility. TPO antibodies are the hallmark of AITD and reflect the severity of the lymphocytic infiltration, regardless of the presence or absence of hypothyroidism. The presence of TPO antibodies increases the risk of developing hypothyroidism during treatment with amiodarone (11).

Our results agree with reports that could not find a relationship between AITD and dosage or duration of amiodarone treatment $(4,7,8,12,13)$. We also could not demonstrate that a baseline cardiac condition such as Chagas' disease was associated with AITD. Silva et al. (14) detected AITD among 83 Chagas' disease patients and found prevalence rates of $28 \%$ for $\mathrm{AlH}$ and of $10 \%$ for AIT, while considering only our 53 Chagas' disease patients, the prevalence rates for AlH were $33.9 \%$ (18 cases) and 5.6\% for AIT (3 cases). Even though our findings were similar considering $\mathrm{AlH}$, we detected at least half the prevalence of AIT, which could reflect differences in population characteristics. Of note, there was a higher incidence of goiter among the Chagas' disease patients studied by Silva et al. (14), while there were no cases of goiter in our group.

The prevalence of clinically overt thyroid disease was strikingly low (32\%) among AlH patients, while the opposite was found for the AIT group (77.7\%), observations that are again consistent with previous reports $(1,4,8,13)$. It should be emphasized that thyroid dysfunction was evaluated by a trained endocrinologist according to formal standards
$(9,15)$. Since AlH is the most prevalent amiodarone-induced thyroid dysfunction, these findings support the recommendation for screening.

The 53 baseline euthyroid patients from the same area without a history of thyroid dysfunction, using a chronically stable dose of amiodarone for more than 6 months, represent a highly selected group, but a very common clinical scenario. The high incidence of AITD should be attributed to the primary effect of the drug on the thyroid instead of an exacerbation of a chronic subclinical disease or a misdiagnosis, which is very common in the early phase of treatment with this drug because of the fluctuations of thyroid hormone levels. Even in this selected population, the incidence of AITD was very high, again further supporting the need for screening.

Recommendations for screening vary depending on the report or guideline, probably reflecting some of the problems previously described, with intervals ranging from 3 months to more than 1 year $(1,4,7)$. If we consider the more clinical scenario represented by our population, it seems reasonable to recommend an interval of 6 months if there are no clinical signs or symptoms of thyroid dysfunction. To recommend a shorter screening interval, we would have to have better information regarding the prognosis of untreated subclinical AITD, which, in the light of available evidence, seems to be very favorable (1), especially for the more prevalent type of AlH.

Our study has some limitations. First, some censoring bias may have been left in our data since we included for analysis only the patients who were being followed at our cardiology outpatient clinic. These could, in theory, represent only the patients who had not suffered any complication with amiodarone prior to inclusion. Another limitation that should be kept in mind concerns other factors that could impact on AITD, such as iodine intake. If this is not kept constant during follow-up, there may be different incidence rates and new screening strategies might be appropriate.

AITD is common even after excluding potential confounders that could be responsible for the high prevalence rates that have been described, which further support the current recommendations for screening. It seems reasonable to recommend screening for thyroid function at 6-month intervals, unless clinical follow-up dictates otherwise or further information regarding the prognosis of untreated subclinical AITD is available.

\section{References}

1. Connolly SJ. Evidence-based analysis of amiodarone efficacy and safety. Circulation 1999; 100: 2025-2034.

2. Kinsara AJ. Amiodarone in the new ACLS guidelines. Circulation 2001; 104: E44.

3. Vorperian VR, Havighurst TC, Miller S, January CT. Adverse effects of low dose amiodarone: a meta-analysis. J Am Coll Cardiol 1997; 30: 791-798.
4. Basaria S, Cooper DS. Amiodarone and the thyroid. Am J Med 2005; 118: 706-714.

5. Martino E, Aghini-Lombardi F, Bartalena L, Grasso L, Loviselli A, Velluzzi $F$, et al. Enhanced susceptibility to amiodarone-induced hypothyroidism in patients with thyroid autoimmune disease. Arch Intern Med 1994; 154: 27222726. 
6. Gheri RG, Pucci P, Falsetti C, Luisi ML, Cerisano GP, Gheri $\mathrm{CF}$, et al. Clinical, biochemical and therapeutical aspects of amiodarone-induced hypothyroidism (AlH) in geriatric patients with cardiac arrhythmias. Arch Gerontol Geriatr 2004; 38: 27-36.

7. Goldschlager N, Epstein AE, Naccarelli G, Olshansky B, Singh B. Practical guidelines for clinicians who treat patients with amiodarone. Practice Guidelines Subcommittee, North American Society of Pacing and Electrophysiology. Arch Intern Med 2000; 160: 1741-1748.

8. Stelfox HT, Ahmed SB, Fiskio J, Bates DW. Monitoring amiodarone's toxicities: recommendations, evidence, and clinical practice. Clin Pharmacol Ther 2004; 75: 110-122.

9. Ladenson PW, Singer PA, Ain KB, Bagchi N, Bigos ST, Levy EG, et al. American Thyroid Association guidelines for detection of thyroid dysfunction. Arch Intern Med 2000; 160: 1573-1575.

10. Stata Statistical Software. [Computer program]. College Station: StataCorp.; 2005.
11. Trip MD, Wiersinga W, Plomp TA. Incidence, predictability, and pathogenesis of amiodarone-induced thyrotoxicosis and hypothyroidism. Am J Med 1991; 91: 507-511.

12. Schaan BD, Cunha CP, Francisconi A, Zottis B, Brum G, Bruch RS, et al. Amiodarone-induced thyroid dysfunction in a tertiary center in south Brazil. Arq Bras Endocrinol Metabol 2005; 49: 916-922.

13. Bartalena L, Wiersinga WM, Tanda ML, Bogazzi F, Piantanida E, Lai A, et al. Diagnosis and management of amiodarone-induced thyrotoxicosis in Europe: results of an international survey among members of the European Thyroid Association. Clin Endocrinol 2004; 61: 494-502.

14. Silva JR, Guariento ME, Fernandes GA, Maciel RM, Ward LS. Impact of long-term administration of amiodarone on the thyroid function of patients with Chagas' disease. Thyroid 2004; 14: 371-377.

15. Maciel LMZ. O exame físico da tireóide. Medicina 2007; 40: 72-77. 